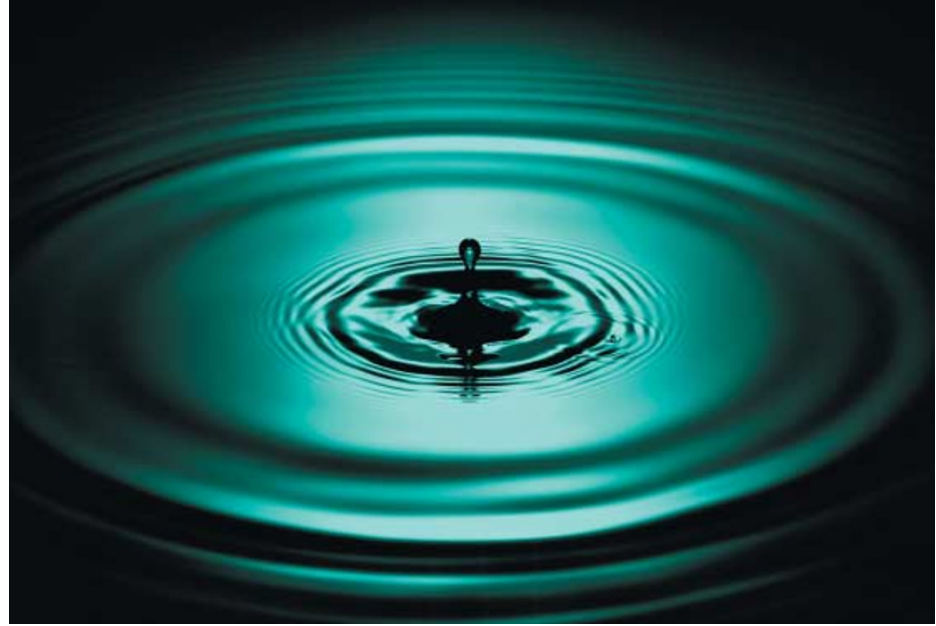

\title{
Small change, large effect
}

Single-nucleotide polymorphisms might be useful in predicting the efficacy of anticancer therapy, but evidence of exactly how these small changes in DNA can have such important consequences is lacking. KyoungJin Sohn and colleagues have focused on the C667T polymorphism in methylenetetrahydrofolate reductase (MTHFR), which is present in about $35 \%$ of the North-American population. They have discovered that the altered enzyme affects the concentration and distribution of folates in cancer cells, which, in turn, alters the chemosensitivity to antifolate drugs.

MTHFR catalyses the conversion of 5,10-methylene THF to 5-methyl THF. The 667T variant of MTHFR is less active, so 5,10-methylene THF accumulates. The authors tested whether the polymorphism altered the effectiveness of the antifolate drugs 5-fluorouracil (5-FU) and methotrexate (MTX) — which are used widely to treat breast and colon cancer - and correlated with their known mechanisms of action.

The authors took a colon cancer and a breast cancer cell line with endogenous 667C MTHFR and transfected them with the $667 \mathrm{~T}$ variant. Enzyme activity was 35\% lower in the 667T MTHFR cells and the intracellular distribution of folates had also changed: levels of 5-methyl THF were about $12 \%$ lower and 5,10methylene THF levels were about $10 \%$ higher. These cells grew faster than $667 \mathrm{C}$ variant cells, probably because 5,10 -methylene THF is required for DNA synthesis.

So, how sensitive are the $667 \mathrm{~T}$ variant MTHFR cells to antifolate drugs? The authors postulated that the effect of 5-FU, which acts by forming an inhibitory complex with thymidylate synthase and 5,10-methylene THF, would be increased by the presence of the polymorphism in cancer cells, and this was the case both in vitro and in xenograft models. By contrast, the authors thought that the effect of MTX, which acts by decreasing levels of 5,10-methylene THF, might be compromised by the polymorphism, and this also proved true for the breast cancer cell line. There was no difference in chemosensitivity to MTX in the $667 \mathrm{~T}$ variant colon cancer cells, but as colon cancer does not usually respond to MTX, this was not that surprising. Interestingly, if MTHFR expression and activity was more completely inhibited by transfecting with antisense to MTHFR, the colon cancer cells were more resistant to MTX.

These data provide evidence of a functional consequence of the C677T MTHFR polymorphism in response to chemotherapy and support the view that this polymorphism might be a useful pharmacogenetic marker for providing tailored chemotherapy to patients with cancer.

Ezzie Hutchinson

\section{(2) References and links} ORIGINAL RESEARCH PAPER Sohn, K.-J. et al. Effect of the methylenetetrahydrofolate reductase C667T polymorphism on chemosensitivity of colon and breast cancer cells to 5-fluorouracil and methotrexate. J. Natl Cancer Inst. 96, 134-144 (2004)

FURTHER READING Ulrich, C. M., Robien, K. \& McLeod, H. L. Cancer pharmacogenetics: polymorphisms, pathways and beyond. Nature Rev. Cancer 3, 912-920 (2003) WEB SITE

Young-In Kim's lab:

http://www.utoronto.ca/nutrisci/faculty/kim.html

\section{TRIAL WATCH}

\section{Debugged}

Chronic Helicobacter pylori infection is associated with gastric cancer, but could its eradication in chronic carriers prevent gastric cancer? Wong et al. performed the first prospective, randomized, placebo-controlled, population-based study to determine whether $H$. pylori eradication in a high-risk population reduced the incidence of gastric cancer.

The study was performed in a high-risk region of southern China and involved 1,630 carriers of $H$. pylori infection - 988 of whom did not have any precancerous lesions, as determined by endoscopic evaluation. H. pylori was eradicated in the participants through combination treatment with omeprazole and antibiotics. After a follow-up of 7.5 years, the authors report that the incidence of gastric cancer was similar among participants who received $H$. pylori eradication treatment and those who received placebo ( 7 and 11 cases, respectively). In those carriers who did not have precancerous lesions, however, eradication of $H$. pylori seemed to prevent the development of gastric cancer - none of the treated patients developed gastric cancer, whereas six in the placebo group did.

Although $H$. pylori eradication seems to be effective in preventing gastric cancer in high-risk populations, further studies are required to determine the effects in low-risk populations, and also to determine the long-term effects of antibiotic treatment in patients with precancerous lesions.

ORIGINAL RESEARCH PAPER Chun-Yu Wong, B. et al. Helicobacter pylori eradication to prevent gastric cancer in a high-risk region of China - a randomized controlled trial. JAMA 291, 187-194 (2004)

\section{Insufficient evidence}

It is a common belief that participating in clinical trials leads to a better outcome for patients with cancer, regardless of whether they are assigned to the control or experimental group of the study. But, researchers at the Dana-Farber Cancer Institute have found little evidence to support this 'trial effect' and advocate that it should no longer be emphasized as an incentive for patient recruitment.

Peppercorn et al. systematically looked for studies that presented primary data comparing the outcome of patients with cancer, within and outside clinical trials. The identified studies were critically evaluated for evidence of a trial effect using a conceptual framework designed by the authors. Only 14 of the 26 identified studies showed evidence of improved outcome for trial participants, but most were unreliable, as they did not effectively control for study bias such as co-morbidity, socio-economic status and performance status. Eight studies compared trial participants with non-trial participants who met the criteria for trial selection, but only three showed improved outcome for trial participants. In addition, the studies showing improved outcome tended to involve children with cancer, patients treated before 1986 and patients with haematological malignancies.

This work indicates that there is insufficient evidence to confirm that the trial effect exists. Even though there might be no immediate benefit from participating in a clinical trial, it is important for trial participants to realize the benefit for future patients.

ORIGinal Research PAPER Peppercorn, J. M., Weeks, J. C., Cook, E. F. \& Joffe, S. Comparison of outcomes in cancer patients treated within and outside clinical trials: conceptual framework and structured review. Lancet 363, 263-270 (2004) 